\title{
DISTRIBUTION OF RABBIT IgG IN THE ACCESSORY SEXUAL GLAND TISSUES OF THE MALE RABBIT
}

\author{
R. J. ABLIN* \\ Division of Immunology, Millard Fillmore Hospital, Buffalo, \\ New York 14209, U.S.A.
}

(Received 15th Fune 1971, accepted 14th September 1971)

\begin{abstract}
Summary. Direct immunofluorescent staining of specimens of accessory sexual gland tissue and of coagulo-prostatic fluid, a normal secretory product of the coagulating and prostate glands of the male rabbit with fluorescein-labelled goat anti-rabbit IgG antiserum revealed localization of IgG within the folds of the submucosal stroma of acini of the coagulating and prostate glands. Binding of IgG to secretory granules within the lumina of these glands and presumably to these same granules seen in preparations of smears of coagulo-prostatic fluid was also seen. Similar immunofluorescent staining of the seminal vesicles disclosed localization of IgG to the stroma and faint intercellular or cytoplasmic fluorescence of glandular epithelial cells.

The possible implications of these findings relative to the development of autoantibodies to accessory sex gland tissues and fluids of the male rabbit and the means of acquisition of IgG by these tissues and fluids are discussed.
\end{abstract}

\section{INTRODUCTION}

Several studies from this and other laboratories have dealt quite extensively with the experimental production of autoantibodies to accessory sexual gland tissues and fluids of the male rabbit as a consequence of parenteral and/or cryo (freezing in situ) stimulation. The results of these studies have been the subject of a recent review (Ablin, Soanes \& Gonder, 1970).

The spectrum of immunological reactions obtained with 'coagulo-prostatic fluid' (CPF) (Ablin, Pfeiffer, Gonder \& Soanes, 1969; Ablin, Bronson \& Soanes, 1970; Ablin, Gonder \& Soanes $1971 \mathrm{~b}$ ) - a normal secretory product of the coagulating and prostate glands of the male rabbit-has been of interest in seeking an explanation for this immune response (Ablin, Gonder \& Soanes, 1971b; Ablin, Witebsky, Jagodzinski \& Soanes, 1971).

In view of the findings of previous immunological studies (Ablin et al., 1969; Ablin, Bronson \& Soanes 1970; Ablin, Gonder \& Soanes 1971a, b) and of a recent report of the distribution of rabbit IgG in the tissues and fluids of the female reproductive tract by Symons \& Herbert (1971), it seemed of

Present address : Immunology Section, Renal Unit, Memorial Hospital of Springfield, Springfield, Illinois 62701 . 
interest to attempt to localize by means of the fluorescent antibody method the possible binding of IgG in vivo in the accessory sex gland tissues of the male rabbit. Localization might elucidate not only the method of transport of IgG into the accessory sex gland secretions of the male, particularly in CPF, but also provide some insight into the nature of the immunological reactions involving GPF.

\section{MATERIALS AND METHODS}

Specimens of rabbit accessory sex gland tissue, i.e. seminal vesicles, coagulating and prostate glands, were removed immediately following exsanguination from mature male New Zealand albino rabbits (weighing 8 to $10 \mathrm{lb}$ ). The specimens were quick-frozen in liquid nitrogen and sectioned at $4 \mu \mathrm{m}$ in a cryostat.

Coagulo-prostatic fluid was obtained from the dorsal-longitudinal sac of freshly extirpated coagulating and prostate glands which were pooled and prepared as described previously (Ablin et al., 1969). Approximately 0.05 ml of CPF was placed on a slide, allowed to spread naturally, and air-dried.

Tissue and fluid specimens were treated (unfixed) with a fluorescein isothiocyanate-conjugated goat antiserum to DEAE-purified rabbit IgG prepared according to the method of Beutner, Sepulveda \& Barnett (1968). This fluorescent conjugate had an apparent fluorescein/protein ratio of 3.56 and was used at $\frac{1}{4}$ unit of $\mathrm{IgG} / \mathrm{ml}$. Elimination of the possibility of cross reactions of this conjugate with the light chains of other immunoglobulin classes was attained through absorption with rabbit Fab fraction (Porter, 1959). Monospecificity was established by immunoelectrophoresis and double diffusion gel precipitation.

Blocking with antiserum to rabbit IgG, untreated with fluorescein, was used as a control for the specificity of the fluorescent staining according to the method of Nairn (1969). An additional control for specificity included treatment of

\section{EXPLANATION OF PLATES 1 AND 2}

Figures 1 to 6 are photomicrographs demonstrating representative examples of the direct immunofluorescent (IF) staining reactions obtained on sections of accessory sex gland tissues and of preparations of smears of coagulo-prostatic fluid (CPF) of the male rabbit following the application of a $1: 32$ dilution of fluorescein-labelled goat antiserum to rabbit IgG.

\section{PLATE 1}

FIGs. 1 and 2. Direct IF staining reactions illustrating the localization of IgG within the folds of the submucosal stroma of acini of the coagulating (Fig. 1) and prostate glands (Fig. 2) and the focal accumulations of secretory granules within the lumina of these acini. $\times 100$.

\section{PLATE 2}

Fig. 3. Section of coagulating gland comparable to that shown in Pl. 1, Fig. 1, illustrating binding of IgG in vivo to accumulations of secretory granules in the lumen. $\times 160$ with oil. FIG. 4. Direct IF staining reaction of smear of CPF, illustrating binding to IgG of secretory granules in vivo. $\times 100$ with oil.

Frg. 5. Direct IF staining of stroma of seminal vesicle. $\times 25$.

Fig. 6. Section of seminal vesicle comparable to that shown in Fig. 5, illustrating faint intercellular or cytoplasmic fluorescence of glandular epithelial cells. $\times 100$ with oil. 

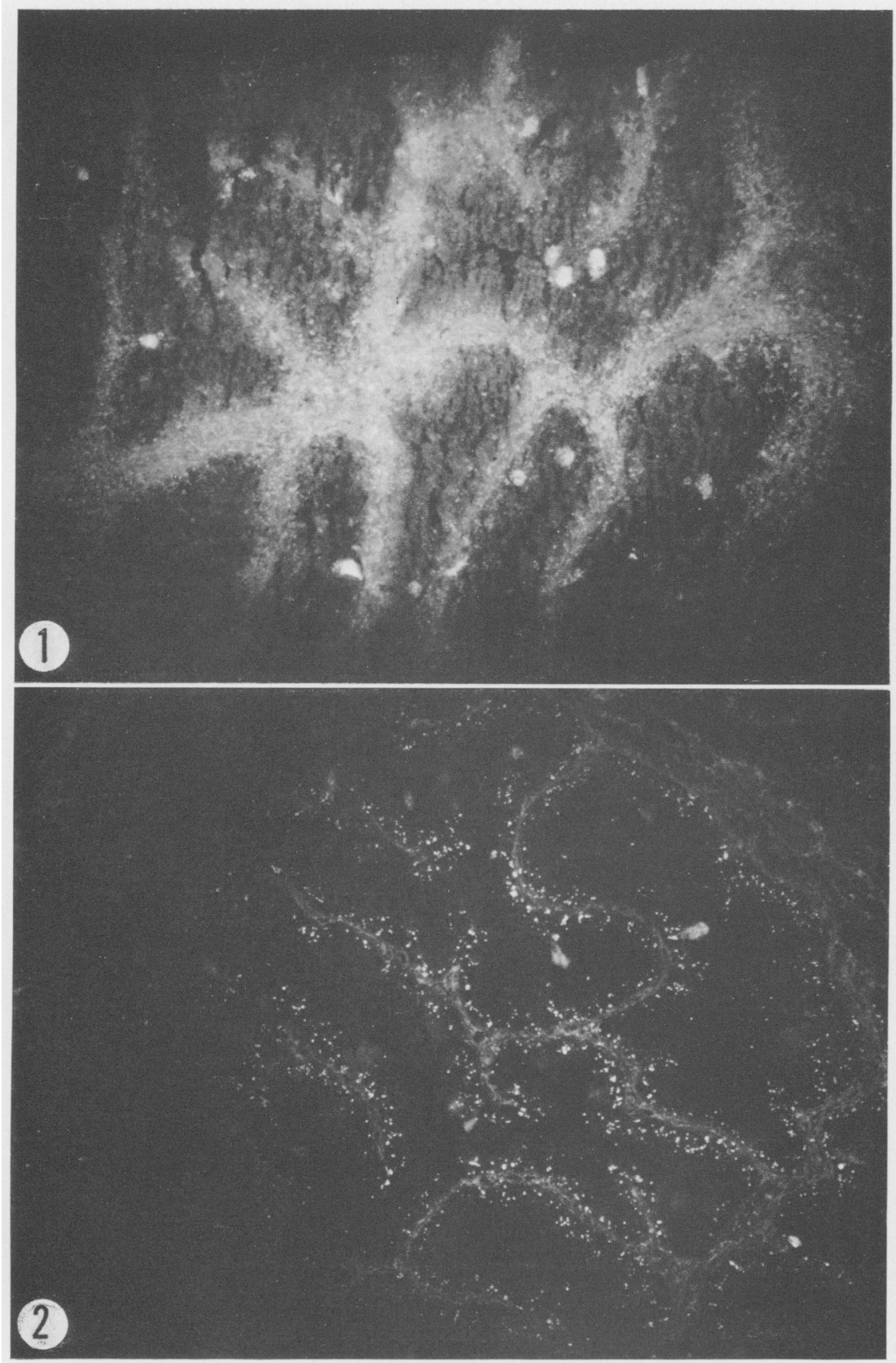

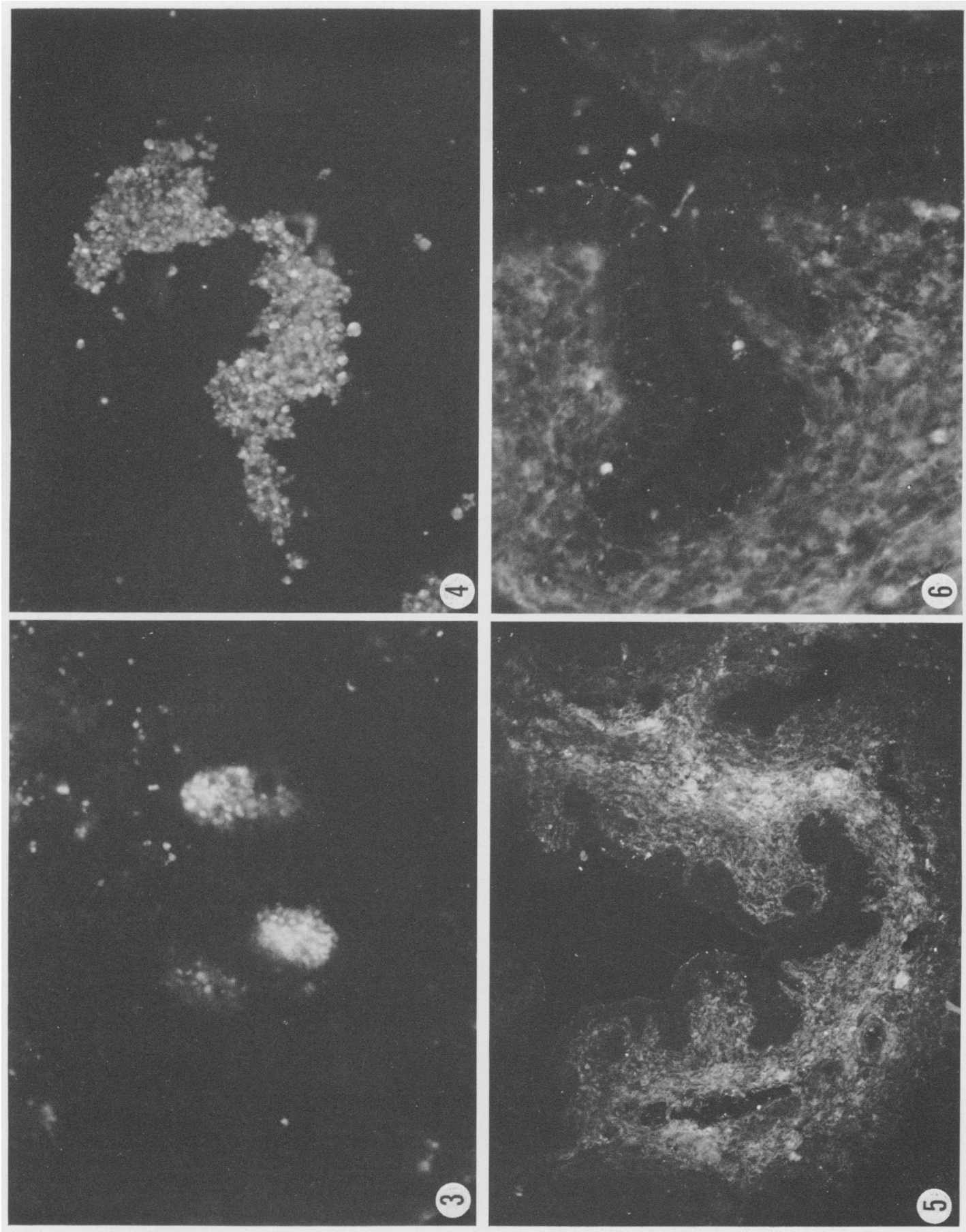
appropriate preparations with a fluorescein-treated goat anti-rabbit IgG serum that had been absorbed with DEAE-purified rabbit $\gamma$-globulin (Cohn Fraction II, Nutritional Biochemical Corp., Cleveland Ohio, Control No. 1281).

\section{RESULTS}

Direct immunofluorescent (IF) staining of specimens of accessory sex gland tissues with fluorescein-labelled goat antiserum to rabbit IgG revealed localization of rabbit IgG as indicated from the moderate binding in vivo observed in a serpentine manner within the folds of the submucosal stroma of acini of the rabbit coagulating and prostate glands (Pl. 1, Figs 1 and 2). Intense binding of IgG to focal accumulations of secretory granules lying within the lumen of coagulating and prostatic tissue acini were seen as shown in Pl. 1, Figs 1 and 2, and P1. 2, Fig. 3.

Immunofluorescent staining of preparations of smears of CPF disclosed a binding of IgG to secretory granules which was identical to that observed within the lumen of sections of coagulating and prostatic tissue (PI. 2, Fig. 4).

Similar staining of sections of rabbit seminal vesicles disclosed moderate to intense binding of IgG to the stroma (Pl. 2, Fig. 5). Examination of such preparations at a higher magnification also revealed (Pl. 2, Fig. 6) intercellular or cytoplasmic fluorescence of glandular epithelial cells.

Treatment of comparable sections of seminal vesicle, coagulating and prostatic tissues and of CPF with unconjugated goat antiserum to rabbit IgG before the application of fluorescein-labelled goat anti-rabbit IgG antiserum markedly reduced the intensity of IF staining. Absorption of the fluoresceinlabelled IgG antiserum with DEAE-purified rabbit $\gamma$-globulin abolished the staining of IgG-containing structures.

\section{DISCUSSION}

Demonstration of the binding of rabbit IgG in the accessory sex gland tissues of the male rabbit in vivo, and the confirmation of the presence of IgG in CPF with the apparent localization of IgG on secretory granules in the lumina of coagulating and prostate gland acini, suggest that the development of an immunological response following freezing of the tissues in situ (Ablin, Witebsky, Jagodzinski \& Soanes, 1971) may be ascribed to the release into the circulation of a potent sequestered immunogen containing IgG, i.e. CPF, as a consequence of damage to the target organs. The potency of GPF may be partly due to its content of autologous $\mathrm{IgG}$ which, as a result of freeze-thaw alteration, may undergo structural modification from its native state to become immunogenic (cf. McCluskey, Miller \& Benacerraff, 1962; Bronson, Ablin \& Soanes, 1970). The mechanism of the immunological response following freezing in situ may be very similar to that occurring in certain autoallergic diseases of the thyroid gland, e.g. Hashimoto's disease, in which, due to a breakdown in tolerance, the escape of thyroglobulin from the thyroid acini leads to the production of autoantibodies (Roitt, Doniach, Campbell \& Hudson, 1956). 
The identical specificity of the antibodies occurring as a result of isoimmunization with CPF (Ablin, unpublished data; Ablin et al., 1971b) to those developed following freezing in situ (Ablin, Soanes \& Gonder, 1970; Ablin, Witebsky, Jagodzinski \& Soanes, 1971) suggests that the primary stimulus for the production of such potent antisera is the result of the parenteral administration of a highly potent and altered (owing to its combination with complete Freund's adjuvant) sequestered immunogen. The development of an immunological response to CPF may be compared with that which develops following inoculation of experimental animals with purified normal thyroglobulin incorporated with complete Freund's adjuvant (Witebsky, Rose, Terplan, Paine \& Egan, 1957).

It is suggested that accessory sex gland tissues and CPF may acquire their content of IgG through a process of transudation from the blood plasma. Confirmation of this hypothesis might be obtained from the identification of antibodies to microbial antigens in CPF following vaccination, as demonstrated in seminal fluid (Katsh \& Katsh, 1965). It is of interest to note that recent IF studies (Ablin et al., 1971a) of normal human prostatic tissue and of prostatic tissue from patients with benign prostatic hypertrophy have revealed localization of $\mathrm{IgG}$ within the basal portion of the cytoplasm of prostatic secretory epithelial cells, and binding in vivo of both IgG and IgA to luminal accumulations of secretory granules. Future IF studies employing antiserum to rabbit IgA may provide further insight into the possibility that secretory epithelial cells of the accessory sex gland tissues of the rabbit are directly involved in the accumulation and discharge of immunoglobulins rather than in their passive acquisition by transudation.

\section{AGKNOWLEDGMENTS}

The author wishes to acknowledge support in part by a grant from the John A. Hartford Foundation, Inc.

\section{REFERENGES}

Ablin, R. J., Bronson, P. \& Soanes, W. A. (1970) Immunoglobulin G: Identification of rabbit IgG in coagulo-prostatic fluid by gel diffusion precipitation and immunoelectrophoresis. Indian $\mathcal{F}$. exp. Biol. 8, 185.

Ablin, R. J., Gonder, M. J. \& Soanes, W. A. (1971a) Localization of immunoglobulins in human prostatic tissue. F. Immun. 107, 603.

Ablin, R. J., Gonder, M. J. \& Soanes, W. A. (1971b) The nature of the immunologic response following in situ freezing of the adnexal glands tissues of reproduction. Cryobiology, 8, 384.

Ablin, R. J., Pfeiffer, L., Gonder, M. J. \& Soanes, W. A. (1969) Precipitating antibody in the sera of patients treated cryosurgically for carcinoma of the prostate. Expl Med. Surg. 27, 406.

Ablin, R. J., Soanes, W. A. \& Gonder, M. J. (1970) Clinical and experimental considerations of the immunologic response to prostatic and other accessory glands tissues of reproduction. Urol. int. 25, 511 .

Ablin, R. J., Witebsky, E., Jagodzinski, R. V. \& Soanes, W. A. (1971) Secondary immunologic response as a consequence of the in situ freezing of rabbit male adnexal glands tissues of reproduction. Expl Med. Surg. 29, 72.

Beutner, E. H., Sepulveda, M. R. \& Barnett, E. V. (1968) Quantitative studies of immunofluorescent staining. Bull. Wld Hlth Org. 39, 587.

Bronson, P., Ablin, R. J. \& SoAnes, W. A. (1970) Evaluation of the freeze-thaw alteration of rabbit male urogenital tissue antigens by double diffusion gel precipitation. Cryobiology, 6, 401 .

Katsh, S. \& Katsh, G. F. (1965) Perspectives in immunological control of reproduction. Pacif. Med. Surg. 73, 28. 
McCluskey, R. T., Miller, F. \& Benacerrafr, B. (1962) Sensitization to denatured autologous gamma globulin. 7. exp. Med. 115, 253.

NAIRN, R. G. (1969) Fluorescent protein tracing. Ed. R. C. Nairn. Williams \& Wilkins, Baltimore.

PORTER, R. R. (1959) The hydrolysis of rabbit $\gamma$-globulin and antibodies with crystalline papain. Biochem. F. 73, 119.

Roitt, I. M., Dontach, D., Gampbell, P. N. \& Hudson, R. V. (1956) Autoantibodies in Hashimoto's disease. Lancet, ii, 820.

Symons, D. B. A. \& HERBERT, J. (1971) Incidence of immunoglobulins in fluids of the rabbit genital tracts and the distribution of IgG-globulin in the tissues of the female tract. F. Reprod. Fert. 24, 55.

Witebsky, E., Rose, N. R., Terplan, K., Paine, J. R. \& Egan, R. W. (1957) Chronic thyroiditis and autoimmunization. F. Am. med. Ass. 164, 1439. 